\title{
Analysis of typical problems and methods in Higher Algebra
}

\author{
Jinzhan Zhang \\ The Department of Mathematics, Longnan Teachers’ College, Chengxian Gansu 742500, China \\ lnszjz1965@163.com
}

Keywords: two type; matrix; polynomial; positive definite matrix

\begin{abstract}
Mathematics has traditionally been a very important subject in the course of learning. It plays a major role in promoting the formation of students' logical thinking and the construction of students' systematic knowledge. As an important part of high school algebra in mathematics to relate people's concern and attention, through examples and methods can make students to learn in the process of clear, prompting students to understand this part. Taking this into account, this paper analyzes and studies the typical problems and methods in advanced algebra, hoping to provide reference for the improvement of algebra teaching quality.
\end{abstract}

\section{Introduction}

When students enter the University, their learning content differs from that of primary and secondary schools, and the knowledge they need is more profound, which brings some difficulties to students' mathematics learning. In particular, algebra of higher mathematics is the most important part of the teaching process. It is very important in the cultivation of students' logical thinking and the learning of students' systematic knowledge. Higher algebra has a certain abstraction, which makes it difficult for many students to understand the essence of the learning process, and it is difficult to grasp the important calculation process. Through the examples of algebraic form for students to explain, with examples of solutions to enable students to master important knowledge, can make students more image based on abstract learning, has the impetus to grasp the students' problem solving method.[1]

\section{The importance of Algebra Learning}

The mathematics learning from a certain aspect, is the study of algebraic algebra, occupy most of the proportion in the whole system of mathematics teaching, which not only affects the performance of students, for students penetration of mathematical thinking, plays an important role in students' mathematical concept of Chengdu. If the entire field of mathematics by analogy become a towering tree, algebra can be said to be the root, through the "roots" growth and winding, we can give the tree adequate nutrition, can promote the tree to grow. Some people once compared algebra to the trunk of huge trees. Various branches of mathematics were branches. Growth of tree trunks, supported the sprouting and sprouting of other branches. No matter, from which point of view, algebra plays an important and fundamental role in the formation of the whole system of mathematics, and it cannot be replaced by other contents. The content of algebra begins with a unary equation, and deepens gradually. It contains so many contexts that algebra is an indispensable part even in the higher level of study in the University and even later". Therefore, the study of algebra cannot be ignored, and the search for the best method of algebra teaching has naturally become an important content for teachers to consider.[2] 


\section{The significance of Higher Algebra Learning for the training of students' comprehensive quality}

Higher algebra and geometry, analysis and other disciplines have a very close relationship, the two are compatible and complementary, in many studies on the cross. This allows students in the course of learning advanced algebra, must not simply stay in the process of learning a single knowledge. Through the study of higher algebra, can let the students knowledge and discipline to be deeper, can let students gradually master the theoretical knowledge and practical ability more. For the cultivation of students' comprehensive quality plays an important role.

\section{Optimize the knowledge of students}

Higher education stage, advanced algebra, analytic geometry, and mathematical analysis are three basic courses, but also a required course for students. In the course of higher algebra teaching, it is necessary to intersect knowledge points such as analytic geometry and mathematical analysis, such as geometry, and also have a solid relationship with them. This enables students to learn not simply to stay in the algebra of a category of learning process, but to achieve a comprehensive grasp of knowledge. Through the algebra of learning, let the students on the relevant knowledge points to be in-depth understanding, through the pertinent knowledge of compaction, for algebra question better solution to provide the path. It is also the integration of a wide range of knowledge that enables highly abstract algebra learning to have a certain image; and the additional knowledge bases required by algebra also enhance the students' comprehensive knowledge[3].

\section{Consolidate the study foundation}

It is a lot of Higher Algebra Learning important foundation, such as "modern algebra", "mathematical modeling", "computation" and "operations research", "mathematics experimental" science cannot do without the knowledge of higher algebra. Only when students master the essential knowledge of advanced algebra can they have a thorough understanding of such courses. Therefore, we must recognize the fundamental role of higher algebra, higher algebra knowledge only through learning, students can study specialized knowledge for the follow-up basis and conditions, to lay the foundation for the better development after[4].

\section{Infiltration of mathematical thinking}

The study of advanced algebra combines many mathematical ideas, such as the combination of figures and shapes, the thought of inversion mapping, the idea of induction and deduction, which are the principal contents that need to be understood in the study of advanced algebra. In the course of Advanced Algebra Learning, students are bound to accept the infiltration of such ideas, and they will inevitably be applied to the course of algebraic questions. Through the study and application of this kind of mathematical thinking, can let the students logical thinking ability and constantly improve, can let the students especially in the learning quality can be established, can make students better image thinking and abstract thinking to be flexible combination. Moreover, through in-depth study of mathematics in algebra, the learning interest of the students will be more and more strong. The mathematics learning initiative will also be improved, has an irreplaceable role in the overall development of students.

\section{The optimization choice and application in Advanced Algebra Teaching}

For the teaching of higher algebra, only through the knowledge teaching and teaching simple, it is difficult to achieve effective teaching effect, therefore, teachers in the process of the teaching of higher algebra, is the need to focus on strategies and countermeasures. Through diverse teaching methods to carry out, make the teaching quality of higher algebra can be improved, so deep into the higher algebra the student's heart, and achieve better application effect.

\section{To cultivate students' interest in learning guided by problems}

Abstract algebra itself is high, its concept, by reference to the theory of logical reasoning, which is subject to restrictions in the form of logic in the teaching process, it is difficult to fully mobilize the enthusiasm of the students. Therefore, $\mathrm{O}$ embarrassed is to pay attention to make the "problem" into 
the algebra teaching process to stimulate the enthusiasm of the students through the problem, let the concept of cognition, students' learning theorem is more efficient, so that students learn to improve the quality[5].

For example, in the process of educating the inverse matrix, the author pays attention to setting up the relevant questions in the form of analogy so that the students can get a thorough understanding of the knowledge. The author is doing the function of the unit matrix in matrix multiplication, which is equivalent to the function of the number 1 in number multiplication, and the inverse matrix of a matrix is equivalent to the reciprocal of a number." The knowledge points in teaching, students will be the first to the reciprocal of the definition to the memory and review, then let students explore the definition of inverse matrix corresponding: if $A$ is a matrix of $N$, if there is a matrix of $n B$, the $A B=B A=E$, then the matrix $A$ is reversible, so $B$ is the inverse of a square matrix $A$. Then the question arises: what kind of matrix is reversible? What kind of matrix is irreversible? How to inverse matrix?

Through the problem of guidance and setting, students can actively participate in classroom teaching, students can participate in the classroom more strongly, and it plays a major role in promoting students' algebra learning. At the same time, teachers in the setting time, also need the consideration of the actual situation of students, and students' interest, starting from the actual situation of students and points of interest is more able to make the problem play a greater role.

Break through the routine and cultivate students' creative thinking

Algebra teaching process, routine teaching process cannot be simply to stay in it too observant of conventional standards., cultivating students' creative thinking in the culture based on the basic knowledge of students, students will form an important initiative, creative thinking, let students get rid of the shackles of the inherent thinking, when new innovation and new ideas to solve the problem, let the students be able to stimulate the consciousness of seeking[6].

For instance, the author pays attention to train students' creative thinking when Clem's law proves the uniqueness of the solution of the system of linear equations.

The coefficient determinant of the $n$ system of linear equations with $n$ equations is:

$$
\mathrm{D}=\left|\begin{array}{cccc}
\mathrm{a}_{11} & \mathrm{a}_{12} & \ldots \ldots & \mathrm{a}_{1 \mathrm{n}} \\
\mathrm{a}_{21} & \mathrm{a}_{22} & \ldots \ldots & \mathrm{a}_{2 \mathrm{n}} \\
\vdots & \vdots & & \vdots \\
\mathrm{a}_{\mathrm{n} 1} & \mathrm{a}_{\mathrm{n} 2} & \ldots \ldots & \mathrm{a}_{\mathrm{nn}}
\end{array}\right| \neq 0
$$

The answer to this question, the algebraic cofactor $\mathrm{k}$ of the coefficient determinant in the linear equation group is needed, $\mathrm{D}_{1 \mathrm{k}}, \mathrm{D}_{2 \mathrm{k}}, \cdots, \mathrm{D}_{\mathrm{nk}}$ is multiplied by the $\mathrm{n}$ equations of the linear equation, and then added. This method of proof has a certain complexity, but it can be simplified by using the method of proof and the property of the determinant.

It is assumed that the solution of a system of linear equations is unique $\left(c_{1}, c_{2}, \ldots, c_{n}\right)$ and $\left(\mathrm{d}_{1}, \mathrm{~d}_{2}, \ldots, \mathrm{d}_{\mathrm{n}}\right)$ two different solutions of linear equations, then $\mathrm{c}_{1}-\mathrm{d}_{1}, \mathrm{c}_{2}-\mathrm{d}_{2}, \ldots, \mathrm{c}_{\mathrm{n}}-\mathrm{d}_{\mathrm{n}}$ at least one of the solutions is not 0 , it can be assumed that $c_{1}-d_{1} \neq 0$

$$
\begin{aligned}
& \text { Because } \sum_{j=1}^{n} a_{i j} c_{j}=b_{i}, \sum_{j=1}^{n} a_{i j} d_{j}=b_{i}, i=1,2, \cdots, n, \\
& \text { So, } i=1,2, \ldots N . \sum_{j=1}^{n} a_{i j}\left(c_{j}-d_{j}\right)=0, i=1,2, \cdots, n
\end{aligned}
$$

The determinant of the nature, it could be concluded that $\left(c_{i}-d_{i}\right) D=0$, and that $\left(c_{i}-d_{i}\right) D \neq 0$ contradictory.

Therefore, if the hypothesis is difficult to establish, it proves the uniqueness of the solution of the system of linear equations of the opposite side. 
The purpose of this question changes the traditional way of proof in the course of resolving the issue, but applies flexibly to the nature of the determinant. It is simpler and more convenient than the conventional method of the higher algebra.

\section{Combination of Figures and forms to train students' intuitions thinking}

Higher algebra has a strong abstraction, which is also an important reason for its difficulty in learning. The use of the combination of figures and shapes to solve all kinds of problems, can improve its simple theory, can make it have a definite sense of space, can make it difficult to reduce learning. Through the application of the combination of figures and shapes, students can learn algebra in a more visual and logical approach in the geometric background, and deepen the understanding of the core ideas in algebra.

For example, in the course of definite integral teaching, the author puts more emphasis on the combination of figures and figures. The definite integral is from curved trapezoid area calculation is introduced, assuming the curve equation is $\mathrm{y}=f(\mathrm{x})$, and $f(\mathrm{x})$ function in the interval [a, b], $f$

$(x) \geqslant 0$, the curved trapezoid style, and take other steps, and the approximate limit calculation process, and the definition of definite integral.

Through the application of this definition, we can obtain the corresponding geometric meaning,

and also can obtain the algebraic $\int_{\mathrm{a}}^{\mathrm{b}} f(\mathrm{x}) \mathrm{dx}$ sum of the trapezoid area at the curved edge.

Through the combination of ideas used to allow students to understand the unbroken image, can also be defined through its references to other auxiliary calculation, so as to reduce the amount of calculation results, to improve students' problem-solving effect[7].

Set up experimental courses to improve students' ability of solving practical problems

The study of mathematics knowledge and the purpose is to solve the practical problems to be analyzed, therefore, teachers in the process of higher algebra teaching, also need to integrate into the experimental curriculum, the curriculum and the experiments provide more practical ability for students, students in the experiment, in the process of practice to enhance the ability of self to solve practical problems let the students find the path of innovation, better in the process of practice, so the study of Higher Algebra value can be fully reflected.

For example, the author carried out in the "Engineering Laboratory", in this laboratory, the author let students incarnate into engineers, combined with the actual situation of engineering, the higher algebra flexible application. In the construction process, given a formula for $y=a x+b$, in the experiment, given a set of $\mathrm{x}$, and a group of $\mathrm{u}, \mathrm{y}$ by $\mathrm{x}$, the application is difficult to accurately calculate $a, b$, this time will be the introduction of higher algebra, least squares method, which can be accurate analysis of all data, and the most likely a, b value. Through this experiment, or small engineering form, let students understand the application value of higher algebra, and let the students try to use knowledge, not only help students to deepen the knowledge, but also help students to find their own weak points, the weak part of the effective promotion and development.

Teachers can also let students use mathematical software to solve the problem, the input and output value of life, these problems can be fully reflected in higher algebra, more can let students understand the applications such as linear equations in time to solve the problem, can let more students to gradually establish mathematical thinking, have important the role for the development of students[8].

\section{Conclusion}

Advanced algebra is the advanced stage of mathematics learning. It has a certain influence and impact on the students' ability. It can improve the students' comprehensive knowledge and skills. Teachers in the process of teaching, it is necessary to pay attention to the use of typical examples, but also focus on one example expand learning ideas of students, so that students can learn to expand the field capacity in higher algebra, let students go into the math ocean, swim. 


\section{References}

[1] Zvyagin N D. Analysis of typical problems that arise in tests of computer software[J]. Measurement Techniques, 2013, 56(4):382-385.

[2]Ortega E, Oñate E, Idelsohn S, et al. A meshless finite point method for three - dimensional analysis of compressible flow problems involving moving boundaries and adaptivity[J]. International Journal for Numerical Methods in Fluids, 2013, 73(4):323 - 343.

[3]Schreiner W N, Surdukowski C, Jenkins R. An approach to the isotypical and solid solution problems in multiphase X-ray analysis[J]. Journal of Applied Crystallography, 1982, 15(6):605 610.

[4]Canha L, Ekel P, Queiroz J, et al. Models and methods of decision making in fuzzy environment and their applications to power engineering problems[J]. Numerical Linear Algebra with Applications, 2010, 14(4):369-390.

[5]Savalei V. CFA with binary variables in small samples: a comparison of two methods[J]. Frontiers in Psychology, 2015, 5:1515.

[6]Ma S, Shi Z, Shao Z, et al. Higher-Order Logic Formalization of Conformal Geometric Algebra and its Application in Verifying a Robotic Manipulation Algorithm[J]. Advances in Applied Clifford Algebras, 2016, 26(4):1-26.

[7]Masoero D, Raimondo A, Valeri D. Bethe Ansatz and the Spectral Theory of Affine Lie Algebra-Valued Connections I. The simply-laced Case[J]. Communications in Mathematical Physics, 2016, 344(3):719-750.

[8]Günaydin M, Skvortsov E, Tran T. Exceptional F, (4) higher-spin theory in AdS 6, at one-loop and other tests of duality[J]. Journal of High Energy Physics, 2016, 2016(11):168. 